\title{
Efeito da Interação Reprodutor x Rebanho na Acurácia da Avaliação Genética de Reprodutores da Raça Holandesa no Brasil
}

\author{
Cláudio Vieira de Araújo², Robledo de Almeida Torres ${ }^{3}$, Cláudio Nápolis Costa ${ }^{4}$, Carmen Silva \\ Pereira $^{3}$, Paulo Sávio Lopes ${ }^{3}$, Ricardo Frederico Euclydes ${ }^{3}$, Rodolpho de Almeida Torres Filho ${ }^{2}$
}

\begin{abstract}
RESUMO - Registros de produção de leite referentes a 37.006 lactações provenientes de 18.540 vacas, filhas de 98 reprodutores e distribuídas em 455 rebanhos de vacas da raça Holandesa foram utilizados com o objetivo de verificar qual seria o efeito nas estimativas dos valores genéticos e na acurácia dessas estimativas, incluindo no modelo o termo de interação reprodutor x rebanho, em comparação com as estimativas de um modelo que não incluía o termo de interação reprodutor x rebanho. A produção de leite foi previamente ajustada para 305 dias de lactação e idade adulta da vaca. Os componentes de variância para produção de leite foram estimados pela máxima verossimilhança restrita, $\mathrm{O}$ teste da razão de verossimilhança foi utilizado na verificação da efetividade da inclusão da interação no modelo. A média da produção de leite foi de $6.936,13 \pm 1.508,36 \mathrm{~kg}$. As estimativas de componentes de variância, obtidas pelo modelo que incluía a interação reprodutor $\mathrm{x}$ rebanho, foram ligeiramente menores do que as dos modelos sem interação, exceto a variância residual, cujas estimativas, nos dois modelos, foram bastante próximas. As estimativas de herdabilidade foram de $0,247 \pm 0,021$ e $0,237 \pm 0,021$, para os modelos com e sem interação reprodutor x rebanho, respectivamente. A proporção da variância fenotípica da produção de leite, devido

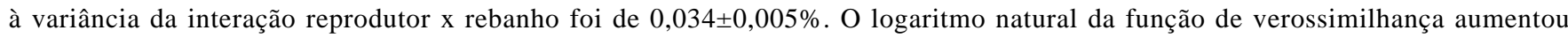
significativamente, quando se incluiu a interação reprodutor $\mathrm{x}$ rebanho no modelo. Os valores genéticos preditos dos reprodutores para a produção de leite ajustada para 305 dias e para idade adulta da vaca foram menores quando o modelo incluiu o termo de interação, embora o valor para a correlação de Spearman tenha sido próximo da unidade.
\end{abstract}

Palavras-chave: avaliação genética, interação reprodutor x rebanho, produção de leite

\section{Effect of the Sire-by-Herd Interaction in the Genetic Evaluation for Milk Yield of Holstein Cows in Brazil}

\begin{abstract}
Yield records of Holstein cows were used to verify the interaction effect on accuracy of sire breeding values. The records of milk yield were adjusted to 305 days of lactation and age of the cow. Variance components for milk yield were estimated using restricted maximum likelihood methodology through two animal models, either not fitting or fitting a sire-by-herd interaction term. Likelihood ratio test was used to verify the effectiveness of including a sire-by-herd interaction effect in the model. Average milk yield was $6,936.13 \pm 1,508.36 \mathrm{~kg}$. The variance components estimates were smaller, when the sire-by-herd interaction was fitted, however, residual variance component, were similar for both models. Heritability estimates for milk yield obtained not fitting and fitting the interaction term in the model, were $0.247 \pm 0.021$ e $0.237 \pm 0.021$, respectivelly. The proportion of the phenotypic variance due to the sire-by-herd interaction variance, was equal to $0.034 \pm 0,005$ percent. The logarithm of the likelihood function was highest fitting a sireby-herd interaction. The breeding values of the sires for 305 day mature milk yield were lower using the model with sire by herd interaction, being of the Spearman correlation value close to unity.
\end{abstract}

Key Words: genetic evaluation, milk yield,sire by herd interaction

\section{Introdução}

A semelhança entre filhas de um touro em um mesmo rebanho reflete tanto a interação genótipo $\mathrm{x}$ ambiente como a covariância ambiental entre o desempenho de meio-irmãs paternas, companheiras de rebanho (MEYER, 1987; COSTA, 1999).

BERESKIN e LUSH (1965), NORMAN (1974) e MEYER (1987) relataram que a covariância ambiental entre meio-irmãs paternas tem origem em fatores biológicos, de manejo e estatísticos, como falha na remoção do efeito de rebanho, de anoestação de parto da vaca ou interação entre rebanhos, de ano, estação e de algum fator fixo, resultando em um modelo inadequado à avaliação genética. Fatores de ajustamento para efeitos fixos também

\footnotetext{
${ }^{1}$ Parte da Dissertação de Mestrado em Zootecnia do primeiro autor, apresentada à UFV, financiada pela CAPES

2 Estudante de Pós-graduação do DZO-UFV. E.mail: cvaraujo@alunos.ufv.br

3 Professor da Universidade Federal de Viçosa - Depto. de Zootecnia, 36570-000, Viçosa-MG.

4 Pesquisador Embrapa Gado de Leite, Rua Eugênio do Nascimento, 610, 36038-330, Juiz de Fora -MG.
} 
podem ser inapropriados a rebanhos específicos. O tratamento diferenciado dado a filhas de um mesmo reprodutor também causa correlação ambiental entre meio-irmãs paternas, visto que filhas de determinado reprodutor são submetidas a um ambiente mais favorável, o que provoca superestimação dos seus valores genéticos.

Se a interação genótipo $\mathrm{x}$ ambiente fosse incluída no modelo de avaliação genética, tanto na forma de covariância ambiental como na forma de interação reprodutor $\mathrm{x}$ rebanho, a influência das observações provenientes de poucos rebanhos seria mais limitada na avaliação genética dos reprodutores. Essa interação poderia não afetar a avaliação genética de animais com progênies em muitos rebanhos, como é o caso de reprodutores utilizados em inseminação artificial, ou poderia ainda não haver alterações no ordenamento dos valores genéticos dos reprodutores. Contudo, ignorar a interação reprodutor $\mathrm{x}$ rebanho implicaria na superestimação das acurácias das avaliações dos reprodutores, principalmente se estes possuíssem progênies distribuídas em poucos rebanhos.

VERDE et al. (1972) analisaram registros de produção de leite, gordura e porcentagem de gordura, em primeiras lactações, de 1.498 vacas da raça Guernsey, 1.250 vacas da raça Holandesa e 2.031 vacas da raça Jersey, filhas de 54, 40 e 61 reprodutores, respectivamente, em que cada reprodutor possuía 10 filhas, pelo menos, em dois diferentes rebanhos. Para produção de leite e gordura, a interação reprodutor $\mathrm{x}$ rebanho respondeu por pequena proporção da variância fenotípica. Para a porcentagem de gordura, a interação respondeu por 0,5 e $14 \%$ da variação total nas raças Holandesa, Jersey e Guernsey, respectivamente.

Utilizando registros de 42.701 em primeiras lactações de vacas Holandesas, filhas de 27 reprodutores usados em inseminação artificial, AHMED (1976) classificou os registros de acordo com o nível de produção dos rebanhos, com a produção para as classes estação-rebanho e com o tamanho dos rebanhos. Médias de quadrados mínimos para os grupos de progênies, agrupados conforme a estratificação das produções, foram calculadas para produção de leite, produção de gordura, produção de proteína, porcentagem de gordura e proteína. A correlação entre os grupos de progênies variou de 0,77 a 0,86 , quando os dados foram classificados de acordo com o nível de produção dos rebanhos; de 0,70 a 0,83 , quando os dados foram classificados de acordo com a produção média por classes rebanho-estação, e de 0,93 a 0,94 , quando os dados foram classificados de acordo com o tamanho de rebanhos. Em nenhuma das estruturas de classificação dos dados ficou evidente a presença da interação reprodutor $\mathrm{x}$ ambiente.

TONG et al. (1977), com vistas em avaliar a importância da interação reprodutor $\mathrm{x}$ rebanho para produção de leite, gordura, proteína, porcentagem de gordura e porcentagem de proteína, utilizaram 13.561 registros de lactações, ajustadas a 305 dias de lactação, de vacas da raça Holandesa. Dois modelos foram empregados; um completo, que continha o efeito da interação reprodutor $x$ rebanho, e outro reduzido, sem $\mathrm{o}$ termo da interação. A interação reprodutor x rebanho respondeu por 4,$1 ; 1,1 ; 0,3 ; 2,6$ e $5,6 \%$ da variação total da produção de leite, gordura, proteína, porcentagem de gordura e porcentagem de proteína, respectivamente. A correlação fenotípica entre as características não foi consideravelmente diferente entre os dois modelos. Embora a correlação genética entre as características tenha sido similar em ambos os modelos, a correlação entre características de produção e características expressas em porcentagem foi maior quando se utilizou o modelo com o termo da interação.

Valores genéticos de reprodutores para produção de leite foram preditos por MOHAMMED et al. (1982) por meio de modelos de reprodutores com e sem interação reprodutor $x$ rebanho e interação reprodutor $\mathrm{x}$ peso ideal da novilha, sendo as interações consideradas aleatórias e nãocorrelacionadas entre si. Esses autores verificaram redução na amplitude dos valores genéticos dos reprodutores, quando algumas das interações foram consideradas no modelo. As correlações de Spearman entre os valores genéticos obtidos por meio dos modelos com e sem interação foram altas, indicando que uma avaliação genética de reprodutores, quando se ignoram as interações reprodutor $\mathrm{x}$ rebanho ou reprodutor $\mathrm{x}$ peso ideal da novilha, não resultaria em maiores alterações no ordenamento dos valores genéticos dos reprodutores.

Por meio de 5.525 registros de produção de leite, oriundos de 48 rebanhos, CHOY e LEE (1991) investigaram o efeito da interação reprodutor $\mathrm{x}$ rebanho sobre a avaliação genética dos animais, mediante modelo animal. Verificaram que o efeito da interação reprodutor $\mathrm{x}$ rebanho sobre a variação total da produção de leite foi relativamente baixo e que o efeito permanente de meio ambiente da vaca foi maior na avaliação genética dos animais, quando comparado ao efeito da interação reprodutor $\mathrm{x}$ rebanho. 
1002 Rev. bras. zootec.

Utilizando dados de produção de leite, gordura e proteína no Reino Unido, referentes às primeiras cinco lactações de 40.066 registros de vacas da raça Holandesa, 17.674 registros de vacas da raça Shorthorn, 7245 registros de vacas da raça Jersey, 19.994 registros de vacas da raça Ayrshire e 16.850 registros de vacas da raça Guernsey, MRODE et al. (1994) verificaram que a proporção da variação total da produção de leite, resultante do componente de variância referente à interação reprodutor x rebanho, variou de 0,024 a 0,063 e de 0,02 a 0,04 , nas primeiras e em todas as lactações, respectivamente. Esses autores concluíram que a interação reprodutor $\mathrm{x}$ rebanho é importante e deve ser considerada na avaliação genética de reprodutores para minimizar vícios.

VAN TASSEL e BERGER (1994), por meio de simulação de dados, examinaram as conseqüências de serem ignorados a interação reprodutor $\mathrm{x}$ rebanho e o parentesco entre reprodutores na estimação do componentes de variância, resultante da interação reprodutor x rebanho, quando a o efeito da interação e foi simulada na estrutura dos dados. Quando o parentesco entre os reprodutores foi ignorado, as variâncias decorrentes do efeito de reprodutor e interação reprodutor $x$ rebanho tenderam à subestimação, à medida que os reprodutores apresentavam parentesco mais próximo. A variância resultante do efeito de reprodutor foi superestimada quando o modelo não incluiu a interação reprodutor $\mathrm{x}$ rebanho, viés que dependeu dos níveis de interação simulados nos dados.

Ao estudar o impacto na avaliação genética de reprodutores, quando foi comparado um modelo que admitia o valor de $2 \%$ da variância total, referente à interação reprodutor $\mathrm{x}$ rebanho, com outro modelo que admitia o valor de $14 \%$ para esta proporção, conforme estabelecido na década de 60, nos Estados Unidos, DIMOV et al. (1996) verificaram que a utilização dos diferentes valores na variância da interação reprodutor x rebanho na avaliação genética causou alterações no ordenamento dos valores genéticos dos animais, embora o valor genético médio de um grupo selecionado de vacas e reprodutores tenha alterado pouco. Esses autores relataram que outros fatores, como, por exemplo, ajustamento para número de ordenhas diárias e ajustamento ao efeito de mês de parto e para o dia do controle, podem ter maiores efeitos na acurácia dos valores genéticos dos animais.
Este estudo teve como objetivo determinar o efeito da interação reprodutor $x$ rebanho sobre a acurácia dos valores genéticos dos reprodutores na avaliação genética de bovinos da raça Holandesa no Brasil.

\section{Material e Métodos}

Os dados utilizados são provenientes do Controle Leiteiro da Associação Brasileira de Criadores da Raça Holandesa e de suas filiadas e compõem o arquivo Zootécnico Nacional de Gado de Leite, sob gerenciamento do Centro Nacional de Pesquisa em Gado de Leite, da Empresa Brasileira de Pesquisa Agropecuária (CNPGL-EMBRAPA), conforme convênio com o Ministério da Agricultura. Os registros constituem 176.399 lactações de 102.718 vacas distribuídas em 1.665 rebanhos, com parições nos anos de 1980 a 1993.

Os dados foram editados para eliminação de registros incompletos, lactações encerradas por causas anormais, lactações inferiores a 150 e superiores a 450 dias, e registros de produção de leite inferiores a $1.000 \mathrm{~kg}$ ou superiores a $15.000 \mathrm{~kg}$. Os meses de parto das vacas foram agrupados em duas épocas, como época 1 , de abril a setembro, e época 2 , de outubro a março. Posteriormente, os efeitos fixos de rebanho, ano e época de parto da vaca foram agrupados em classes de rebanho-ano-época.

Com referência à informação sobre ascendência e origem das vacas, classificaram-se os animais em Grupo 1, Holandês puro de origem, e Grupo 2, Holandês puro por cruzamento, composto de animais com composição genética igual ou superior a 31/32 da raça Holandesa.

Os registros de produção de leite total foram previamente ajustados para duração da lactação e para classes ordem-idade da vaca no parto, por meio de fatores gerados a partir da própria estrutura dos dados.

$\mathrm{O}$ arquivo usado para análise foi restringido à apresentar, no mínimo, quatro observações por classe de rebanho-ano-época no parto, e ainda, impôs-se a restrição de que os reprodutores possuíssem, no mínimo, dez filhas em pelo menos dois diferentes rebanhos, fato que resultou em 37.006 lactações provenientes de 18.540 vacas, filhas de 98 reprodutores e distribuídas em 455 rebanhos.

$\mathrm{O}$ arquivo de pedigree que originou a matriz de numeradores dos coeficientes de parentesco (NRM), utilizada em todas as análises, foi obtido do arquivo de 108.551 lactações mais o pedigree complementar de 615 reprodutores, resultando em 63.409 dados no 
arquivo de pedigree, 96.559 animais diferentes na NRM, 245.800 elementos não-zero na NRM e 709 animais endogâmicos, com coeficiente médio endogâmico de 0,055 .

A análise para predição dos valores genéticos dos animais, considerando-se a inclusão do termo de interação reprodutor $\mathrm{x}$ rebanho, foi obtida por meio do modelo:

$$
\mathbf{y}=\mathbf{\sim} \underset{\sim}{\boldsymbol{\beta}}+\mathbf{Z}_{\mathbf{a}}^{\mathbf{a}} \underset{\sim}{\mathbf{a}}+\mathbf{Z}_{\mathbf{p}} \mathbf{p}+\mathbf{Z}_{\mathbf{s x h}} \underset{\sim}{\mathbf{s}}+\underset{\sim}{\mathbf{e}},
$$

em que: $\mathbf{y}$ é um vetor $\mathrm{n}$ x 1, de $\mathrm{n}$ observações de produção de leite; $\mathbf{X}$ é uma matriz $\mathrm{n} \mathrm{x}$, de incidência de $\mathrm{f}$ níveis dos efeitos fixos; $\beta$ é um vetor $\mathrm{f} x 1$, de efeitos fixos referentes às classes de rebanho-anoépoca no parto e ao grupo genético dos animais; $\mathbf{Z}_{\mathbf{a}}$ é uma matriz $\mathrm{n} \times \mathrm{N}$, de incidência dos valores genéticos; a é um vetor $\mathrm{N} \mathrm{x} \mathrm{1,} \mathrm{de} \mathrm{valores} \mathrm{genéticos} \mathrm{dos}$ animais; $\mathbf{Z}_{\mathbf{p}}$ é uma matriz $\mathrm{n} \times \mathrm{N}$, de incidência dos efeitos permanentes de meio ambiente sobre os animais; $\underset{\sim}{\mathbf{p}}$ é um vetor $\mathrm{N} \times 1$, de valores referentes ao efeito permanente de meio ambiente dos animais; $\mathbf{Z}_{\mathbf{s x h}}$ é uma matriz de dimensão nxh, de incidência dos efeitos da interação reprodutor $\mathrm{x}$ rebanho, composta de zeros e uns; $\underset{\sim}{\mathbf{S}}$ é um vetor h $\mathrm{x}$ 1, de valores referentes ao efeito da interação reprodutor x rebanho; e é um vetor de resíduos da mesma dimensão de $\underset{\sim}{\mathrm{y}}$, sendo:

N Númerodeindivíduos;

n Número total de observações;

f Número de classes de efeitos fixos; e

h Número de níveis da interação reprodutor $x$ rebanho.

As pressuposições acerca da distribuição dos vetores $\mathbf{y}, \mathrm{a}, \mathbf{p}, \mathrm{s}$ e e podem ser descritas como

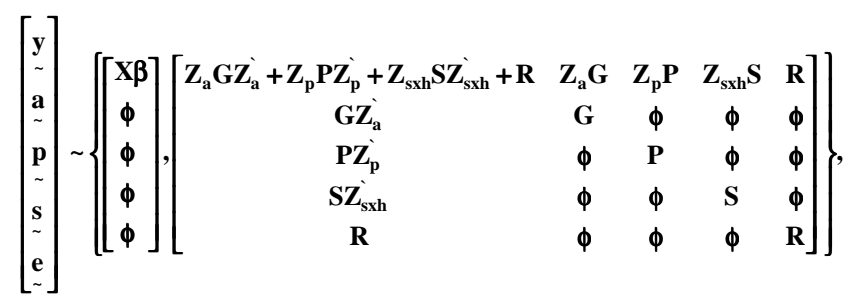

em que:

$$
\begin{aligned}
& \mathbf{G}=\mathbf{A} \cdot \sigma_{\mathrm{a}}^{2} ; \\
& \mathbf{P}=\mathbf{I}_{\mathbf{N}} \cdot \sigma_{\mathrm{p}}^{2} ; \\
& \mathbf{S}=\mathbf{I}_{\mathbf{h}} \cdot \sigma_{\mathrm{sxh}}^{2} ; \\
& \mathrm{R}=\mathrm{I}_{\mathrm{n}} \cdot \sigma_{\mathrm{e}}^{2} ;
\end{aligned}
$$

em que: A = Matriz de numeradores do coeficiente de parentesco entre os indivíduos, de ordem igual ao número total de indivíduos $(\mathrm{N}) ; \sigma_{\mathrm{a}}^{2}=$ Variância genética aditiva para produção de leite; $\mathbf{I}_{\mathbf{n}}=$ Matriz identidade, de ordem $N ; \sigma_{p}^{2}=$ Variância referente ao efeito de ambiente permanente sobre o animal, na produção de leite; $\mathbf{I}_{\mathbf{h}}=$ Matriz identidade, de ordem $\mathbf{h}$; $\boldsymbol{\sigma}^{2}{ }_{\mathbf{s x h}}=$ Variância referente à interação reprodutor $\mathrm{x}$ rebanho, na produção de leite; $\mathbf{I}_{\mathbf{n}}=$ Matriz identidade, de ordem n; e $\sigma_{\mathrm{e}}^{2}=$ Variância residual para produção de leite.

As equações do modelo misto (3.4) são descritas como:

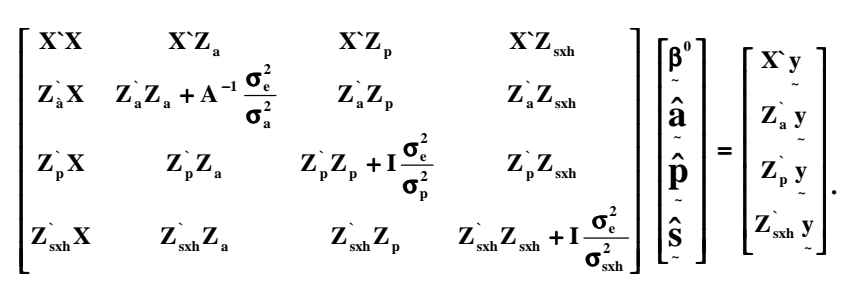

A análise para predição dos valores genéticos dos animais, desconsiderando-se a inclusão do termo de interação reprodutor $x$ rebanho, foi obtida por meio de um modelo similar ao modelo 1.1, excluindo-se, no entanto, o termo de interação reprodutor x rebanho

As acurácias $\left(\mathrm{r}_{\mathrm{ti}}\right)$ das estimativas dos valores genéticos foram obtidas por meio da seguinte expressão:

$$
r_{\mathrm{ti}}=\sqrt{1-\frac{\mathrm{PEV}}{\sigma_{\mathrm{a}}^{2}}},
$$

em que: PEV a variância do erro de predição, estimado por

$$
\operatorname{PEV}=\left(\mathrm{z}_{\mathrm{a}}^{\prime} \mathrm{Z}_{\mathrm{a}}+\mathrm{A}^{-1} \frac{\sigma_{\mathrm{e}}^{2}}{\sigma_{\mathrm{a}}^{2}}\right)^{-1}
$$

As estimativas de componentes de (co)variâncias e predições dos valores genéticos dos animais foram obtidas pelo programa MTDFREML (Multiple Trait Derivative Free Restricted Maximum Likelihood), descrito por BOLDMAN et al. (1995), em que se utiliza a máxima verossimilhança restrita livre de derivadas na estimação de componentes de variância e o sistema de equações de modelos mistos na predição de valores genéticos dos animais. $\mathrm{O}$ critério adotado na convergência da variância dos valores do simplex foi de, no mínimo, $10^{-9}$.

Para determinar a importância da inclusão do efeito da interação reprodutor $\mathrm{x}$ rebanho no modelo de avaliação genética dos animais, utilizou-se o teste da razão de verossimilhança de modelos 
1004 Rev. bras. zootec.

seqüencialmente reduzidos (RAO, 1973). A estimativa da estatística do teste da razão de verossimilhança (LR) foi comparada com o valor obtido por meio da distribuição de qui-quadrado, com 1 grau de liberdade e nível de significância fixo em $1 \%$.

A correlação de Spearman entre os valores genéticos preditos dos reprodutores para a produção de leite ajustada para 305 dias de lactação e idade adulta, incluindo-se ou não no modelo o termo de interação reprodutor $\mathrm{x}$ rebanho, foi obtida do pacote estatístico Statistical Analisys System (SAS, 1990).

\section{Resultados e Discussão}

A média e o desvio-padrão observados na produção de leite, ajustada para um período de lactação de 305 dias e idade adulta da vaca, foi igual a 6.936,13 \pm $1.508,36 \mathrm{~kg}$. Estimativas de componentes de variância, herdabilidades, proporção da variância fenotípica decorrente do efeito permanente de meio ambiente da vaca $(\mathrm{C} 1)$ e proporção da variância fenotípica decorrente do efeito da interação reprodutor $\mathrm{x}$ rebanho (C2), quando consideradas no modelo, bem como valores do logaritmo natural da função de verossimilhança, para modelos sem e com o termo de interação reprodutor x rebanho, são apresentadas na Tabela 1 .

Ao incluir o termo de interação reprodutor $x$ rebanho no modelo estatístico, verifica-se que houve reduções das estimativas de componentes de variância referentes ao efeito genético aditivo e ao efeito de ambiente permanente, as quais foram de, aproximadamente, 4 e $10 \%$, respectivamente. A variância residual praticamente não se alterou com a inclusão da interação no modelo. Reduções nas estimativas de componentes de variância, quando o modelo inclui a interação reprodutor x rebanho, foram observadas por TONG et al. (1977) e MEYER (1987).

A estimativa do componente de variância, referente ao efeito da interação reprodutor $\mathrm{x}$ rebanho, foi relativamente baixa. Algum viés poderia ser encontrado nessa estimativa, uma vez que desprezou-se parentesco entre os reprodutores, subestimando o componente de variância.

Essa subestimação, no entanto, deve ser relativamente baixa, uma vez que há pouca perda de informação ao desconsiderar o parentesco entre os reprodutores, já que o nível do efeito da interação reprodutor $\mathrm{x}$ rebanho é relativamente baixo. VAN TASSEL e BERGER (1994), ao verificarem o efeito de se desprezar o parentesco entre reprodutores na estimação dos componentes de variância, em razão
Tabela 1 - Estimativas de componentes de variância resultante do efeito genético aditivo $\left(\hat{\sigma}_{a}^{2}\right)$, efeito permanente de meio ambiente da vaca $\left(\hat{\sigma}_{\mathrm{C}^{1}}^{2}\right)$, efeito da interação reprodutor $x$ rebanho $\left(\hat{\sigma}_{\mathrm{C}^{2}}^{2}\right)$, efeito residual $\left(\hat{\sigma}_{\mathrm{e}}^{2}\right)$, herdabilidades, proporções da variância fenotípica resultante do efeito permanente de meio ambiente da vaca $\left(\hat{\mathrm{C}}^{1}\right)$, efeito da interação reprodutor $\mathrm{x}$ rebanho $\left(\hat{\mathrm{C}}^{2}\right)$, efeito residual (ê).

Table 1 - Additive genetic $\left(\hat{\sigma}_{\mathrm{a}}^{2}\right)$, environment uncorrelated $\left(\hat{\sigma}_{\mathrm{C}^{1}}^{2}\right)$, sire by herd interaction $\left(\hat{\sigma}_{\mathrm{C}^{2}}^{2}\right)$ and residual $\left(\hat{\sigma}_{\mathrm{e}}^{2}\right)$ variance component estimates for 305 day mature equivalent milk yield, estimates of heritability $\left(h^{2}\right)$, proportion of the variance phenotypic regarding variance of the permanent environment effect $\left(C^{1}\right)$, proportion of the variance phenotypic regarding variance of the sire by herd effect $\left(C^{2}\right)$ and proportion of the variance phenotypic regarding variance of the residual effect (ê)

\begin{tabular}{lcc}
\hline $\begin{array}{l}\text { Componentes } \\
\text { de variância }\end{array}$ & \multicolumn{2}{c}{ Modelos } \\
Variance & \multicolumn{2}{c}{ Models } \\
\cline { 2 - 3 } components & Sem interação & Com interação \\
& Without interaction & With interaction \\
\hline & 341841,4260 & 328591,0303 \\
& 265010,4688 & 238126,6705 \\
& - & 47443,0331 \\
Parâmetros & 776527,7308 & 774549,1055 \\
Parameters & & \\
& $0,247 \pm 0,021$ & $0,237 \pm 0,021$ \\
& $0,192 \pm 0,018$ & $0,171 \pm 0,018$ \\
& - & $0,034 \pm 0,005$ \\
& $0,561 \pm 0,008$ & $0,558 \pm 0,008$ \\
\hline
\end{tabular}

da interação reprodutor $\mathrm{x}$ rebanho, observaram que, embora o componente de variância tenha sido subestimado quando o parentesco entre reprodutores foi ignorado, este viés foi relativamente baixo, quando os dados representavam populações que utilizavam inseminação artificial. Dessa forma, segundo eles, o parentesco entre os reprodutores poderia ser ignorado, com pequena perda de informação, quando esses reprodutores não fossem parentes próximos, ou quando o nível do efeito de interação reprodutor $\mathrm{x}$ rebanho não fosse elevado. BANOS e SHOOK (1990), por meio de modelo de reprodutor, também não consideraram o parentesco entre reprodutores na estimativa do componente de variância, em virtude da interação reprodutor $x$ rebanho na produção de leite e da contagem de células somáticas. Esses autores afirmaram que o vício esperado seria de pequena escala, pois o parentesco entre reprodutores era de pequena proporção.

Como conseqüência da pequena diferença das estimativas de componentes de variância entre os 
dois modelos, as estimativas de herdabilidade e as proporções da variância fenotípica, resultante do efeito permanente de meio ambiente, do efeito da interação reprodutor $\mathrm{x}$ rebanho e da variância ambiental, foram próximas entre si, quando se consideram os erros-padrão das estimativas.

O componente de variância, referente à interação reprodutor $x$ rebanho, representou somente $3,4 \%$ da variância total da produção de leite, ajustada para 305 dias de lactação e idade adulta. Este resultado é similar ao observado por MEYER (1987) ao utilizar o modelo de reprodutor para produção de leite, em primeiras lactações. BANOS e SHOOK (1990) observaram valores de $1,84,2,11$ e 3,00 \%, para primeira, segunda e terceira ordens de parto, respectivamente. DIMOV et al. (1995) encontraram, em rebanhos provenientes da Califórnia, valores de 1,5 $\%$ para a primeira lactação e de $1,9 \%$ para a primeira, segunda e terceira lactações em conjunto, e valores de 1,5 e $1,8 \%$ para a primeira e para as demais lactações, respectivamente, em rebanhos oriundos de Nova York e Pensilvânia. KELLEHER et al. (1967), LEE (1976) e TONG et al. (1977) verificaram proporções da variância total, devidas à covariância ambiental entre meio-irmãs paternas, que variaram de 2 a $10 \%$, estimadas pelos métodos I e III, de Henderson (HENDERSON, 1984).

A inclusão da interação reprodutor $\mathrm{x}$ rebanho no modelo não provocou redução considerável da variância residual, contudo, esse efeito, quando considerado na análise, provocou aumento significativo $(\mathrm{P}<0,01)$ no logaritmo natural da função de verossimilhança, conforme indicado pelo teste da razão de verossimilhança para modelos seqüencialmente reduzidos, cujo valor foi 64,0854 , o que indica a superioridade do modelo, quando se inclui a interação. MEYER (1987) verificou também maiores valores para os logaritmos das funções de verossimilhança em modelos que apresentavam interações reprodutor $\mathrm{x}$ rebanho e reprodutor $\mathrm{x}$ rebanho-ano-estacão de parto, em relação ao modelo que desconsiderou essas interações.

As médias, os desvios-padrão, os valores mínimos e máximos e as amplitudes, para valores genéticos preditos com suas acurácias, quando os reprodutores são avaliados pelos modelos sem e com termo de interação reprodutor $\mathrm{x}$ rebanho, são apresentados na Tabela 2. O valor médio dos valores genéticos dos reprodutores, para produção de leite ajustada para 305 dias de lactação e idade adulta da vaca, foi menor quando o modelo incluiu o termo de interação, embora os desvios-padrão tenham sido bem maiores que os valores médios. O mesmo comportamento pode ser observado para as amplitudes dos valores genéticos. Essa redução da amplitude dos valores genéticos, quando se inclui a interação reprodutor $\mathrm{x}$ rebanho no modelo, conduz à menor discriminação entre os reprodutores.

$\mathrm{O}$ alto valor para a correlação de Spearman (Tabela 2) confirma que praticamente não se esperam diferenças entre o ordenamento dos reprodutores quanto aos seus valores genéticos, quando se utiliza o modelo com ou sem inclusão da interação reprodutor $\mathrm{x}$ rebanho.

A interação reprodutor $\mathrm{x}$ rebanho referida na forma de interação genótipo $\mathrm{x}$ ambiente ou de covariância ambiental, procura quantificar a similaridade entre filhas de um reprodutor em um mesmo

Tabela 2 - Médias, desvios-padrão, valores mínimos e máximos e amplitudes dos valores genéticos preditos $(\hat{\mathrm{g}})$, erros-padrão de predição (EP) e acurácias (Rti) dos reprodutores avaliados

Table 2 - Means, standard deviation, minimum and maximum sire breeding value estimates $(\hat{\mathrm{g}})$ with standard errors (EP) and accuracy (Rti)

\begin{tabular}{|c|c|c|c|}
\hline $\begin{array}{l}\text { Modelos } \\
\text { Models }\end{array}$ & $\hat{\mathrm{g}}$ & $\mathrm{EP}$ & Rti \\
\hline $\begin{array}{l}\text { Sem interação } \\
\text { Without interaction }\end{array}$ & & & \\
\hline $\begin{array}{l}\text { Média }(\mathrm{kg}) \\
\text { Average }(\mathrm{kg})\end{array}$ & 224,1437 & 178,3675 & 0,9486 \\
\hline $\begin{array}{l}\text { Desvio-padrão }(\mathrm{kg}) \\
\text { Standard deviation }(\mathrm{kg})\end{array}$ & 457,9814 & 41,8637 & 0,0232 \\
\hline $\begin{array}{l}\text { Mínimo (kg) } \\
\text { Minimum (kg) }\end{array}$ & $-920,8440$ & 88,7000 & 0,87 \\
\hline $\begin{array}{l}\text { Máximo }(\mathrm{kg}) \\
\text { Maximum }(\mathrm{kg})\end{array}$ & $1.456,5800$ & 289,6800 & 0,99 \\
\hline $\begin{array}{l}\text { Amplitude (kg) } \\
\text { Range }(k g)\end{array}$ & $2.377,4240$ & 200,98 & 0,12 \\
\hline $\begin{array}{l}\text { Com interação } \\
\text { With interaction }\end{array}$ & & & \\
\hline $\begin{array}{l}\text { Média }(\mathrm{kg}) \\
\text { Average }(\mathrm{kg})\end{array}$ & 207,6270 & 189,6854 & 0,9408 \\
\hline $\begin{array}{l}\text { Desvio-padrão }(\mathrm{kg}) \\
\text { Standard deviation }(\mathrm{kg})\end{array}$ & 436,2188 & 41,4368 & 0,0261 \\
\hline $\begin{array}{l}\text { Mínimo }(\mathrm{kg}) \\
\text { Minimum }(k g)\end{array}$ & $-893,3270$ & 98,3800 & 0,85 \\
\hline $\begin{array}{l}\text { Máximo }(\mathrm{kg}) \\
\text { Maximum }(\mathrm{kg})\end{array}$ & $1.361,1600$ & 299,4500 & 0,99 \\
\hline $\begin{array}{l}\text { Amplitude }(\mathrm{kg}) \\
\text { Range }(\mathrm{kg})\end{array}$ & $2.254,4870$ & 201,07 & 0,14 \\
\hline $\begin{array}{l}\text { Correlação de Spearma } \\
\text { Spearman correlation }\end{array}$ & an 0,997 & 0,981 & \\
\hline
\end{tabular}


1006 Rev. bras. zootec.

rebanho. Ao ignorar-se a interação reprodutor $\mathrm{x}$ rebanho houve aumento da amplitude dos valores genéticos preditos dos reprodutores e, como conseqüência, aumento do componente de variância genética aditiva, resultando em menores valores para a variância do erro de predição e, conseqüentemente, ligeira superestimação da acurácia da maioria dos valores genéticos preditos dos reprodutores. $\mathrm{O}$ impacto dessa superestimação torna-se mais severo em avaliações de reprodutores cujas progênies estão localizadas em poucos rebanhos, uma vez que esses reprodutores teriam as acurácias de seus valores genéticos preditos superestimadas.

\section{Conclusões}

Não houve qualquer alteração importante no ordenamento dos valores genéticos dos reprodutores, quando se inclui no modelo a interação reprodutor $\mathrm{x}$ rebanho. Ao ignorar-se a interação reprodutor $\mathrm{x}$ rebanho, superestima-se o componente de variância genético aditivo. Essa superestimação não foi maior devido ao fato de que os reprodutores possuem progênies distribuídas em vários rebanhos. Assim, a distribuição dos reprodutores nos rebanhos, bem como o número de filhas distribuídas nestes rebanhos, é que irá determinar a necessidade de incorporar no modelo estatístico a interação reprodutor $\mathrm{x}$ rebanho.

\section{Agradecimento}

À Associação Brasileira de Criadores de Bovinos da Raça Holandesa e ao Centro Nacional de Pesquisa de Gado de Leite da EMBRAPA, pelo fornecimento dos dados utilizados nesta pesquisa.

\section{Referências Bibliográficas}

AHMED, M.K.A. 1984. Sire by level of production interaction in dairy cattle. World Review of Anim. Prod., 20(4):9-13.

BANOS, G., SHOOK, G.E. 1990. Genotype by environment interaction and genetic correlations among parities for somatic cell count and milk yield. J. Dairy Sci., 73(9):2563-2573.

BOLDMAN, K.G., KRIESE, L.A., VAN VLECK, L.D. et al. 1995. A manual for use of MTDFREML: a set of programs to obtain estimates of variances and covariances (DRAFT). Lincoln: Department of Agriculture/Agriculture Research Service. $125 \mathrm{p}$.
CHOY, Y.S., LEE, K.J. 1992. Effects of permanent environment and herd-sire interaction on the model. Korean J. Anim. Sci., 34(6):327-333.

COSTA, C. N. Interação genótipo x ambiente em gado de leite. In: SIMPÓSIO INTERNACIONAL DE GENÉTICA E MELHORAMENTO ANIMAL, 1, 1999, Viçosa. Anais ...Viçosa: UFV, 1999. p.161-191.

DIMOV, G., ALBUQUERQUE, L.G., KEOWN, J.F. et al. 1995. Variance of interaction effects of sire and herd for yields traits of Holsteins in California, New York and Pennsylvania with an animal model. J. Dairy Sci., 78(4):939-946.

DIMOV, G., KEOWN, J.F, VAN VLECK, L.D. et al. 1996. Effect of variance of interaction effects of sire and herd on seletion for milk and fat yield. J. Dairy Sci., 79(1):140-144.

HENDERSON, C.R. 1984. Aplication of linear models in animal breeding. University of Guelf. 462p.

MAO, I.L., BURNISIDE, E.B. 1969. Sire by herd environment interaction for milk production. J. Dairy Sci., 52(7):105-1062.

MEYER, K. 1987. Estimates of variance due to sire $\mathrm{x}$ herd interactions and environmental covariances between paternal half-sibs for first lactation dairy production. Livest. Prod. Anim. Sci., 17(2):95-115.

MOHAMMAD, W.A., LEE, A.J., GROSSMAN, M. 1982. Genotype-environment interaction in sire evaluation. Journal of Dairy Science, 65(5):857-860.

MRODE, R.A, SWANSON, G.J.T., SMITH, C. et al. Animal model estimates of sire-herd interactions for production traits for the major dairy breeds in the United Kingdom. In: WORLD CONGRESS ON GENETICS APPLIED TO LIVESTOCK PRODUCTION, 5, 1994, Ontario. Proccedings ...Ontario, 1994. v.17, p.19-22.

RAO, C.R. 1973. Linear statistical inference and its aplications. 2.ed., New York: John Wiley e Sons. 552p.

STATISTICAL ANALISYS SYSTEM INSTITUTE. 1990. SAS User's Guide: Statistics version 6, fourth edition. Cary: SAS Intitute Inc. 1686p.

TONG, A K.W., KENNEDY, B.W., MOXLEY, J.E. 1977. Sire by herd interactions for milk yield and composition traits. Can. J. Anim. Sci., 57(3):383-388.

VAN TASSELL, C.P, BERGER, P.J. 1994. Consideration of sire relationships for estimation of variance components with interaction of herd and sire. J. Dairy Sci., 77(1):313-324,

VERDE, O.G., WILCOX, C.J., MARTIN, F.G. et al. 1972. Sire by herd interaction for milk production in Florida DHIA herds. Flórida: Flórida University. 19p.

Recebido em: 06/09/00

Aceito em: 12/03/01 